\title{
A hybrid image similarity measure based on a new combination of different similarity techniques
}

\author{
Nisreen Ryadh Hamza1 ${ }^{1}$, Rasha Ail Dihin ${ }^{2}$, Mohammed Hasan Abdulameer ${ }^{3}$ \\ ${ }^{1}$ Faculty of Computer Science and Information Technology, University of Qadisiyah, Iraq \\ ${ }^{2,3}$ Department of Computer Science, Faculty of Education for girls, University of Kufa, Iraq
}

\begin{tabular}{l}
\hline Article Info \\
\hline Article history: \\
Received May 9, 2019 \\
Revised Oct 25, 2019 \\
Accepted Oct 31, 2019 \\
\hline
\end{tabular}

\section{Keywords:}

Feature similarity index measure FSIM

Gaussian noise

Histogram similarity measure

HSSIM

Salt and pepper noise

SSIM

Uniform noise

\section{Corresponding Author:}

Mohammed Hasan Abdulameer, Department of Computer Science, Faculty of Education for Girls, University of Kufa, Iraq.

Email: mohammed.almayali@uokufa.edu.iq

\begin{abstract}
Image similarity is the degree of how two images are similar or dissimilar. It computes the similarity degree between the intensity patterns in images. A new image similarity measure named $(H F E M M)$ is proposed in this paper. The HFEMM is composed of two phases. Phase 1, a modified histogram similarity measure (HSSIM) is merged with feature similarity measure $(F S I M)$ to get a new measure called (HFM). In phase 2, the resulted (HFM) is merged with error measure $(E M M)$ in order to get a new similarity measure, which is named (HFEMM). Different kindes of noises for example Gaussian, Uniform and salt \& pepper noise are used with the proposed methods. One of the human face databases $(A T \& T)$ is used in the experiments and random images are used as well. For the evaluation, the similarity percentage under peak signal to noise ratio (PSNR) is used. To show the effectiveness of the proposed measure, a comparision anong different similar technique such as SSIM, HFM, EMM and HFEMM are considered. The proposed HFEMM achieved higher similarity result when PSNR was low compared to the other methods.
\end{abstract}

Copyright () 2020 Institute of Advanced Engineering and Science. All rights reserved.

\section{INTRODUCTION}

Image similarity in recent years has become a key point in image processing applications for example monitoring, restoration and other applications [1]. Similarity can be characterized as the contrast between two images, and the similarity measure is a numerical difference between two dissimilar images under comparison [2]. When the two images match up to the maximum similarity, the similarity degree between two signals is required to test the system and in order to make a decision [3]. Similarity measure methods can be classified into: information theoretical techniques and statistical techniques [4]. Several studies related to similarity techniques have been presented recently. In 1995, proposed a new informationtheoretic approach (Mutual Information) [5]. In 2004, the researchers presented a scale called (SSIM) based on the information of the structure [6]. In 2014, introduced a new measure called (HSSIM) that based on 'joint histogram'. The measure outperforms statistical measure (SSIM) [7]. On the other hand, in 2017, the researchers proposed measure similarity called (NMSE) depened on normalized mean square error [8]. A New likeness measure based on 'Affinity Propagation' introduced in 2018 [9]. As well, in 2018 introduced likeness measure based on "joint histogram-Entropy" [10]. In 2019, intrduoced ahybrid measure for similarity of images [11]. In this paper, proposed a hybrid measure for image similarity called (HFEMM). The rest of this paper is organized as follow: the similarity techniques is presented in section 2, the research method is explained in section 3, section 4 presents the results and discussions and the conclusions is presented in section 5 . 


\section{SIMILARITY TECHNIQUES}

Techniques that used for Similarity can be classified into: the statistical and the information theoretical techniques $[4,7]$.

\subsection{Statistical similarity techniques}

It is possible to obtain valuable information from the image via calculating 'statistical measurements' for example 'Mean', 'Variance' and (SD) where SD means standerd divation. This information can be utilized to calculate similarity of image [12].

\subsubsection{Structural similarity measure (SSIM)}

In 2004, the authors introduced a new statistical measure for image quality index called Structural Similarity Index Method (SSIM) $[6,13]$ that utilized distance function to measurement the likeness relied upon statistical features [12]. The measure can be display in (1):

$$
\operatorname{SSIM}(p, q)=\frac{\left(2 \mu_{p} \mu_{q}+C 1\right)\left(2 \sigma_{p q}+C 2\right)}{\left(\mu^{2}+\mu^{2} q^{+}+C 1\right)\left(\sigma^{2}+\sigma^{2}{ }^{2}+C 2\right)}
$$

Where $\mu \mathrm{p}, \mu \mathrm{q}$ are the 'means' and $\sigma^{2}{ }_{\mathrm{p}}, \sigma_{\mathrm{q}}^{2}$ are the 'variance'; $\sigma_{\mathrm{pq}}$ is the 'covariance', $C 1$ and $C 2$ are constants $\left(C 1=(R I P)^{2}, C 2=(R 2 P)^{2}, R 1\right.$ and $R 2$ are constants, $P$ is maximum pixel value $)$.

\subsubsection{Feature similarity measure (FSIM)}

Feature Similarity Measure is a statistical measurement of image quality estimation. FSIM offered by [14]. FSIM computation consists of two phases: the first phase calculates the similarity of local $\left(S_{L}\right)$ as follows:

$$
S_{L}\left(x_{1}\right)=\left[S_{p c}\left(x_{1}\right)\right]^{\alpha} \times\left[S_{G}\left(x_{1}\right)\right]^{\beta}
$$

Where $S_{P c}$ represents the Phase Congruency similarity

$$
\begin{aligned}
& S_{P C}\left(x_{1}\right)=\frac{2 P C_{1}\left(x_{1}\right) \times P C_{2}\left(x_{1}\right)+R 1}{P C_{1}^{2}\left(x_{1}\right) \times P C_{2}^{2}\left(x_{1}\right)+R 2} \\
& P C\left(x_{1}\right)=\frac{R\left(x_{1}\right)}{\epsilon+\sum n A_{n}\left(x_{1}\right)}, R(X 1)=\sqrt{K 1^{2}\left(x_{1}\right)+H} 1^{2}\left(x_{1}\right), A_{n}\left(x_{1}\right)=\sqrt{\left[e_{n}^{2}\left(x_{1}\right)+o_{n}^{2}\right]}
\end{aligned}
$$

Where

$$
H 1\left(x_{1}\right)=\sum_{n} O_{n}\left(x_{1}\right), K 1\left(x_{1}\right)=\sum_{n} e_{n}\left(x_{1}\right), O_{n}\left(x_{1}\right)=\varepsilon\left(x_{1}\right) * M_{n}{ }^{e}, e_{n}\left(x_{1}\right)=\varepsilon\left(x_{1}\right) * M_{n}^{o}
$$

$S_{G}$ represents the GM similarity

$$
\begin{aligned}
& S_{G}\left(x_{1}\right)=\frac{2 G_{1}\left(x_{1}\right) \times G_{2}\left(x_{1}\right)+R 1}{{G_{1}{ }^{2}\left(x_{1}\right) \times G_{2}{ }^{2}\left(x_{1}\right)+R 2}} \\
& G=\sqrt{G_{p}{ }^{2}\left(x_{1}\right)+G_{q}{ }^{2}\left(x_{1}\right)}
\end{aligned}
$$

The second phase is to calculate the FSIM between $F 1(x)$ and $F 2(x)$ :

$$
\operatorname{FSIM}\left\{\mathrm{F} 1\left(\mathrm{x}_{1}\right), \mathrm{F} 2\left(\mathrm{x}_{1}\right)\right\}=\Phi\left\{\mathrm{F} 1\left(x_{1}\right), \mathrm{F} 2\left(\mathrm{x}_{1}\right)\right\}=\frac{\sum_{\mathrm{x}} \in \Omega \mathrm{s}_{1}\left(\mathrm{x}_{1}\right) \times \mathrm{pc}\left(\mathrm{x}_{1}\right)}{\sum_{\mathrm{x}} \in \Omega \mathrm{pc} \mathrm{m}_{\mathrm{m}}\left(\mathrm{x}_{1}\right)}
$$

Where $P C_{\max }\left(x_{1}\right)$ is MAXimum (MAX) between ' $P C_{1}\left(x_{1}\right)^{\prime}$ and ' $P C_{2}\left(x_{1}\right)^{\prime}[15]$.

\subsection{Information-theoretic similarity techniques}

Information-theoretical similarity technique is used to get the similarity depended on intensity values $[16,17]$

\subsubsection{Histogram similarity measure}

Histogram Similarity Measure is the measurement that depends on information theoretical technique via using conventional histogram and common histogram $\mathrm{H}_{\mathrm{ij}}$ [7]. HSSIM suggested previously as SSIM 
scale cannot be well-implemented under signifcant noise. In (HSSIM) the researcher applied the common histogram and combined it with the conventional histogram as follows [18, 19]:

$$
L(p, q)=\sqrt{\frac{\sum_{i} \sum_{j}\left[\left(H_{i j}-H_{j i}\right) \frac{1}{h_{i}+C 1}\right]^{2}}{2 L^{2}}}
$$

Where, $h_{i}$ is the conventional histogram and $C 1$ is a constant. $\mathrm{L}(\mathrm{p}, \mathrm{q})$ can be normalize by using $L 1(p, q)$ which represent the maximum value of the error estimate in very low PSNR as follows:

$$
L L(p 6, q 6)=\frac{L(p, q)}{L 1(p, q)}, r 1(p, q)=1-L L(p 6, q)
$$

\section{RESEARCH METHOD}

The merhod and proposed Measure (HFEMM) is depicted in Figure 1.

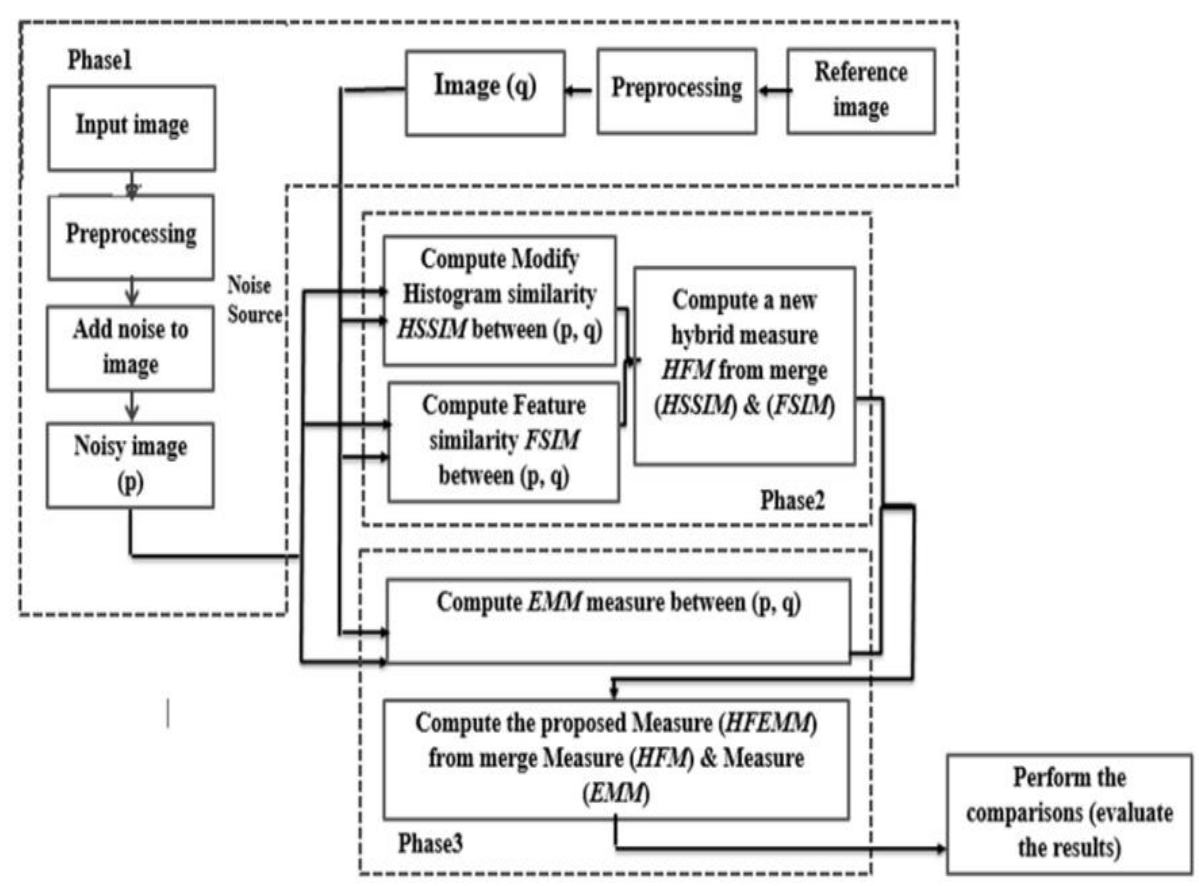

Figure 1 . The similarity between two images (verification) by the proposed similarity measure

\subsection{Phase one}

In this phase, preprocessing performed on both images (refrence and input) to prepare the images. Furthermore, different kinds of noise are added to the input image from a variety of sources [20, 21, 22].

\subsection{Phase two}

In this phase, Modify Histogram Similarity measure is merged with Feature Similarity measure to get a new hybrid measure $(H F M)$ as in (11):

$$
\operatorname{HFM}(p, q)=\sqrt{H(p, q) K+\operatorname{FSIM}(p, q)(1-K)}
$$

$\mathrm{H}(\mathrm{p}, \mathrm{q})$ is Histogram Similarity measure but with a simple change as follows:

$$
H 1(p, q)=\sqrt{\sum_{i} \sum_{j}\left[\left(H_{i j}-H_{j i}\right) \frac{1}{h_{i}+c 1}\right]^{2}}
$$


$H 1(p, q)$ can be normalize by using $H 2(p, q)$ which represent the maximum value of the error estimate in signifcant noise as follows:

$$
H H(p, q)=\frac{H 1(p, q)}{H 2(p, q)} \quad, H(p, q)=1-H H(p, q)
$$

$\operatorname{FSIM}(p, q)$ is Feature Similarity Measure as in equation (8 ).K is very small constant.

\subsection{Phase three $s$}

The other measure is the Error Mean Measure $\operatorname{EMM}(p, q)$ between image $p$ and $q$ (derived from Mean Square Error [23]) as follows:

$$
E M M=1-\left[\frac{1}{\mathrm{NM}} \sum_{\mathrm{n}=0}^{\mathrm{N}-1} \sum_{\mathrm{m}=0}^{\mathrm{M}-1}[\mathrm{p}(\mathrm{n}, \mathrm{m})-\mathrm{q}(\mathrm{n}, \mathrm{m})]^{2} /\left(\sum_{n=0}^{N-1} \sum_{m=0}^{M-1} q(n, m)\right)^{\frac{1}{2}}\right]
$$

By combining the resulted measure from phase two $(H F M)$ and the Error Mean measure $(E M M)$, we will get a new similarity measure called (HFEMM). The (15) is summaries the new measure as form:

$$
\operatorname{HFEMM}(p, q)=(\operatorname{HFM}(p, q)(1-U)+\operatorname{EMM}(p, q)(U))^{1 / 2}
$$

Where $\mathrm{U}$ is very small constant, $0 \leq \operatorname{HFEMM}(p, q) \leq 1$. Finally, perform the comprasions. Algorithm (1) shows the method and proposed measure (HFEMM) for similarity.

\section{Algorithm (1) HFEMM}

Inputs: $\mathrm{p}$ isrefrence image and $\mathrm{q}$ is noisy image, $\mathrm{K}$ and $\mathrm{U}$ are very small constant Outputs: a number between 0 and 1 that represent the similarity.

- Step 1: transform the colour images in to grayscale images.

- Step 2: transform the images into double.

- $\quad$ Step 3: Compute $H$

$$
H 1(p, q)=\sqrt{\sum_{i} \sum_{j}\left[\left(H_{i j}-H_{j i}\right) \frac{1}{h_{i}+c 1}\right]^{2}}
$$

- $\quad$ Step 4: Set $H 2(p, q)=H 1(p, q)$ when noise is maximum.

- Step 5: Normalization: $H H=H 1 / H 2$.

- $\quad$ Step 6: Set $H=1-H H$.

- $\quad$ Step 7: Compute FSIM

$$
\operatorname{FSIM}(p, q)=\frac{\sum_{\mathrm{x} \in \beth} S_{L}(x) \cdot P C_{\max }(x)}{\sum_{\mathrm{x} \in \beth} P C_{\max }(x)}
$$

- $\quad$ Step 8: Compute HFM

$$
H F M=\sqrt{H(p, q) \times K+F S I M(p, q) \times(1-K)}
$$

- $\quad$ Step 9: Compute EMM

$$
\operatorname{EMM}(p, q)=1-\left[\frac{1}{\mathrm{IJ}} \sum_{\mathrm{n}=0}^{\mathrm{N}-1} \sum_{\mathrm{m}=0}^{\mathrm{M}-1}[\mathrm{p}(\mathrm{n}, \mathrm{m})-\mathrm{q}(\mathrm{n}, \mathrm{m})]^{2} /\left(\sum_{n=0}^{N-1} \sum_{m=0}^{M-1} q(n, m)\right)^{\frac{1}{2}}\right]
$$

- $\quad$ Step 10: Compute HFEMM

$$
\operatorname{HFEMM}(p, q)=(\operatorname{HFM}(p, q)(1-U)+\operatorname{EMM}(p, q)(U))^{1 / 2}
$$

- $\quad$ Step 11: Perform the comparisons (evaluate the results)

- $\quad$ Step 12: End of Algorithm 


\section{RESULTS AND DISCUSSIONS}

The proposed meaures implemented using 'MATLAB' environment and a human face database called AT and T was used in testing the proposed methods. Moreover, different kinds of random image are also used for the evaluation. The range of similarity measures among ( 0 and1). If value is (1), then its displays the ideal match between the images, Else if its value is $(0)$, then there is no match between images [24].

\subsection{AT\&T data base}

The AT\&T is an American Telephone \& Telegram: Laboratories from Cambridge comprises a set of various human faces images were taken in (April 1992 and April 1994) at the database lab. It comprises of 10 dissimilar images (poses) of every person, image size $(92 \times 112)$ pixels [25].

\subsection{Evaluation the proposed measure on AT\&T dataset}

This first of evaluation includes implementing the similarity measure under different kinds of noise such as Gaussians, salt and pepper and uniform noise. Diffent types of images from AT and T database are adopted and tested, for example an image as in the Figure 2 below was tested using Gaussians noise. Table 1 and Figure 3 are show the similarity results between the proposed measure and other measures such as (SSIM, EEM, HFM) under Gaussian noise.

In addition, the second test shows the implementation under salt and pepper noise as shown in Figure 4. Table 2 and Figure 5 shown similarity result between the proposed measure and another measures unde salt $\&$ pepper noise. So as to show the steadiness and adequacy of the proposed measure, we applied the proposed measure under a combination of noises (Gaussian and Uniform noise, Gaussian and salt and pepper noise) which will be more noisy, and As show in the follwing Figure 6. The Figure 7 shows the performance under Gaussian noise and slat and pepper noise Table 3 shown similarity result between the proposed measure and another under Gaussian and Uniform noise. Table 4 show the similarity result between the proposed measure and another under Gaussian and salt and pepper noise.

\subsection{Evaluation the proposed measure by using different kinds of images}

In this section, we used different kinds of image to display the effectiveness and efficiency of that the proposed measure. As illustrated in Figures 8-10. Comparisons of similarity measures under Gaussian and uniform noise a shown in Figure 11
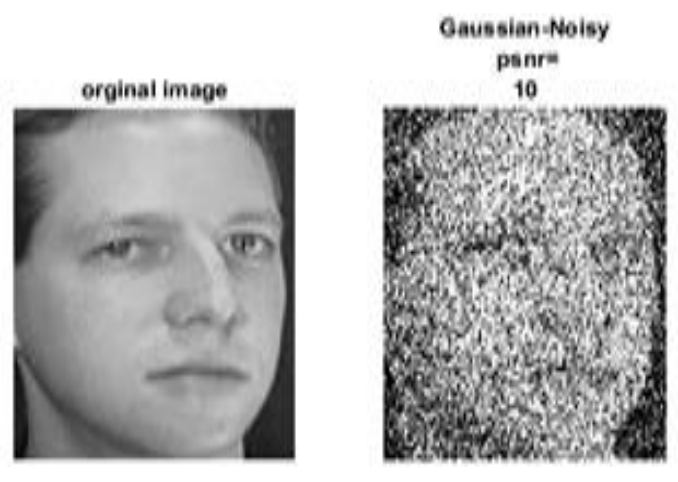

Figure 2. Original image and noisy image with Gaussian noise

Table 1. Comparisons of similarity measures for same images under Gaussian noise

\begin{tabular}{ccccc}
\multicolumn{5}{c}{ (Higher noise ratio 100\% when psnr $=100$ ) } \\
\hline Ratio of Noise & SSIM & EMM & HFM & HFEMM \\
\hline 65\% (PSNR-50) & 0.0020 & 0.0001 & 0.3072 & 0.4293 \\
52\% (PSNR-30) & 0.0020 & 0.0008 & 0.2990 & 0.4465 \\
38\% (PSNR-10) & 0.0034 & 0.0830 & 0.3924 & 0.5383 \\
32\% (PSNR 0) & 0.0141 & 0.2155 & 0.5605 & 0.6891 \\
28\% (PSNR 10) & 0.0633 & 0.5469 & 0.7871 & 0.8824 \\
1\% (PSNR 50) & 0.9831 & 0.9999 & 0.9996 & 1.0000 \\
\hline
\end{tabular}



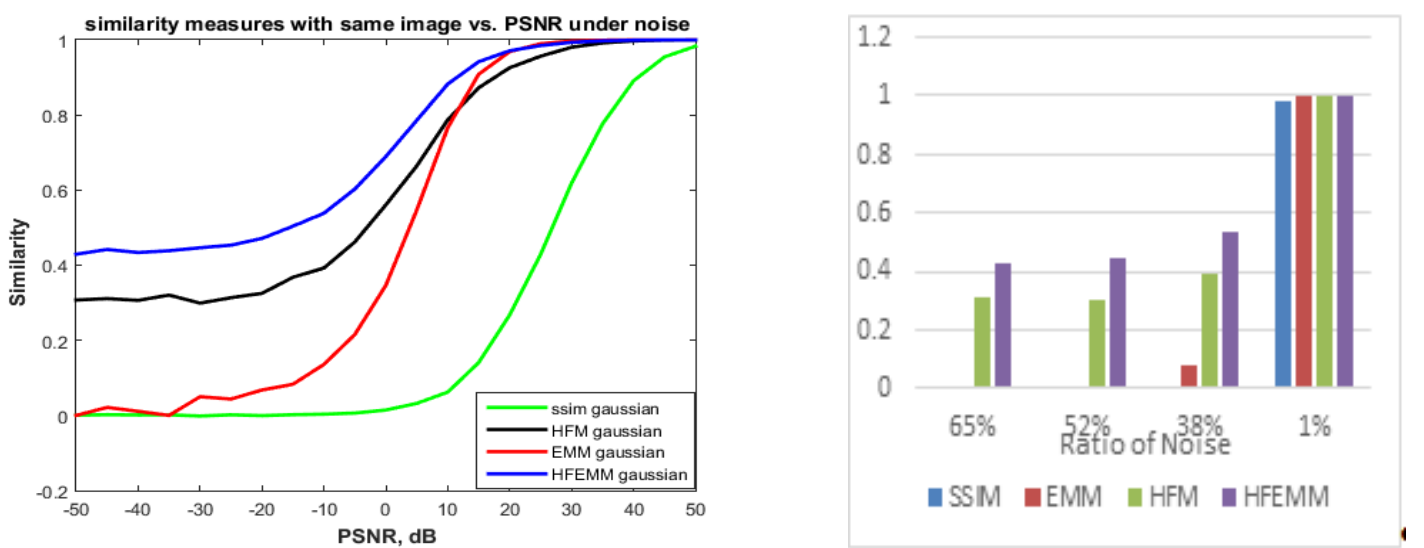

Figure 3. Comparisons of similarity measures for same imagesu under Gaussian noise
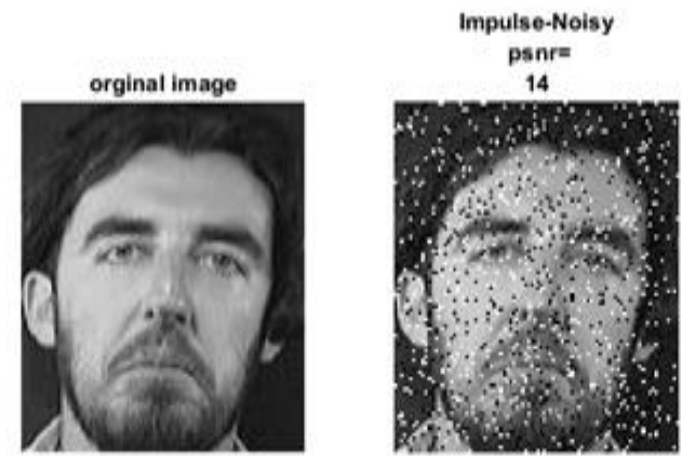

Figure 4. Original image and noisy image with salt and pepper noise

Table 2. Comparisons of similarity measures for same images under salt and pepper noise

\begin{tabular}{ccccc}
\hline Ratio of Noise & SSIM & $E M M$ & $H F M$ & HFEMM \\
\hline 27\% (PSNR 9.9650) & 0.0930 & 0.6511 & 0.7735 & 0.8512 \\
22\% (PSNR 15.3765) & 0.2963 & 0.8972 & 0.9176 & 0.9536 \\
$18 \%$ (PSNR 20.4279) & 0.5835 & 0.9676 & 0.9716 & 0.9849 \\
16\% (PSNR 23.7130) & 0.7289 & 0.9848 & 0.9854 & 0.9925 \\
$12 \%$ (PSNR 28.8983) & 0.9067 & 0.9954 & 0.9957 & 0.9978 \\
8\% (PSNR 32.4168) & 0.9527 & 0.9984 & 0.9983 & 0.9991 \\
\hline
\end{tabular}
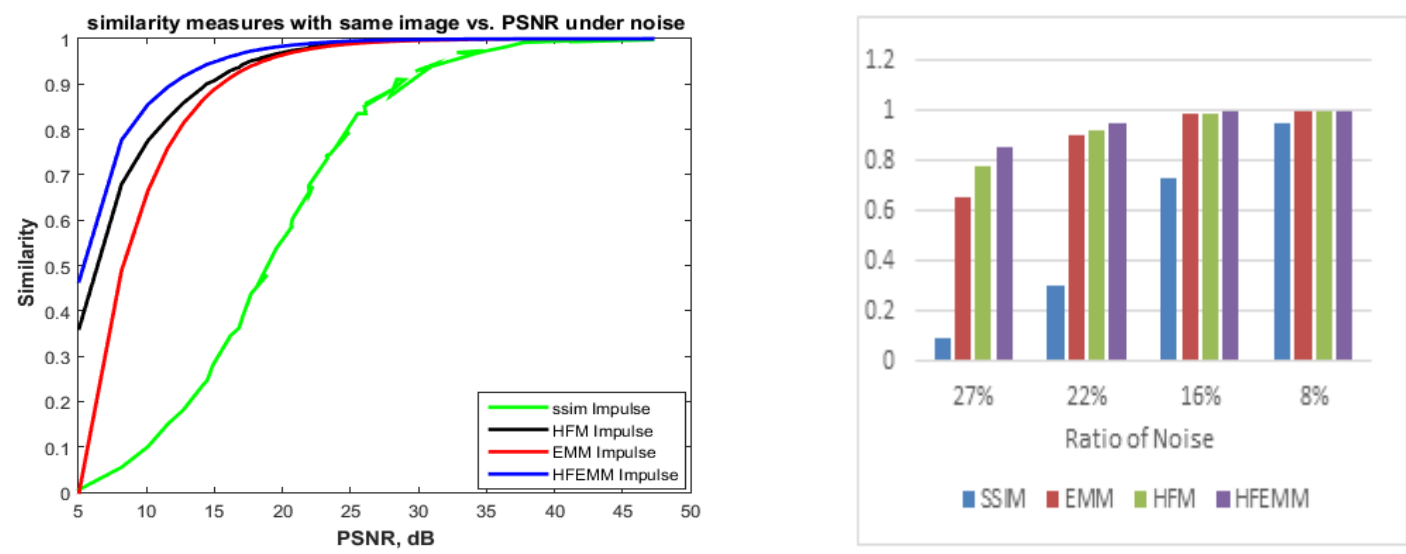

Figure 5. Comparisons of similarity measures for same images under impulse noise 

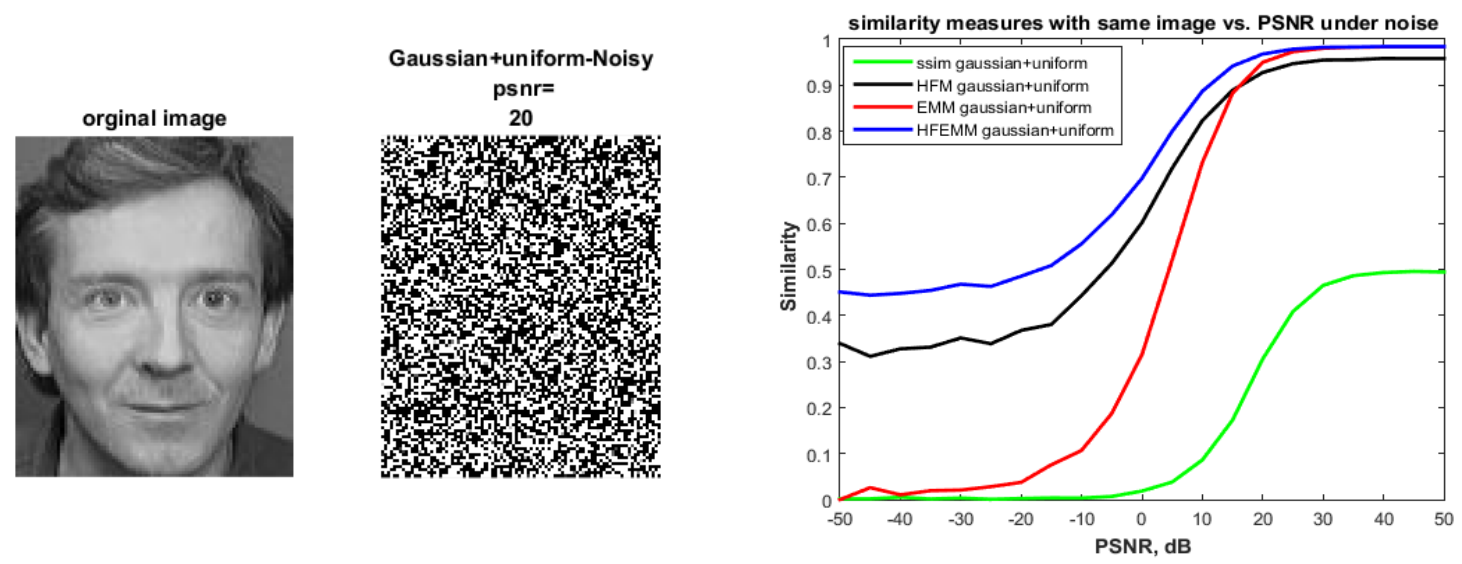

Figure 6. (P) Original image and noisy image (q) comparisons of similarity measures under Gaussian+uniform noise
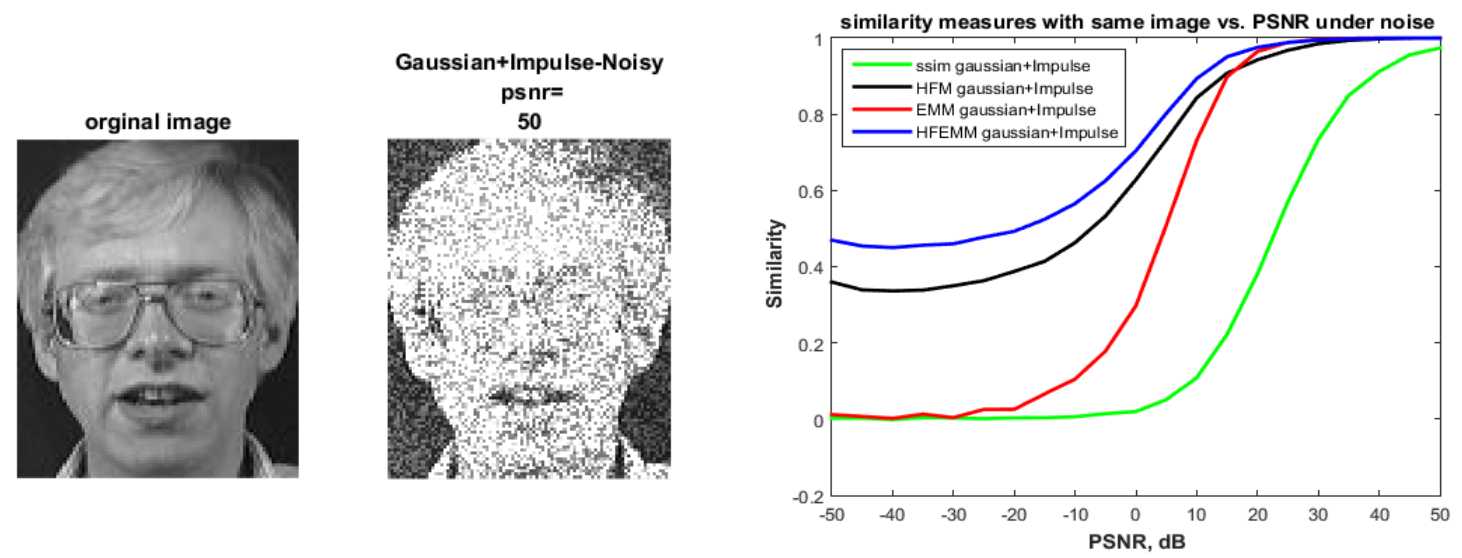

Figure7. (P) original image and noisy image (q) comparisons of similarity measures under gaussian+salt and pepper noise

Table 3. Comparisons of similarity measures for same images under Gaussian and uniform noise

\begin{tabular}{ccccc}
\hline Ratio of Noise & SSIM & $E M M$ & $H F M$ & HFEMM \\
\hline 65\% (PSNR-50) & 0.0005 & 0.0000 & 0.3391 & 0.4510 \\
52\% (PSNR -30) & 0.0000 & 0.0207 & 0.3510 & 0.4679 \\
38\% (PSNR -10) & 0.0053 & 0.1069 & 0.4431 & 0.5555 \\
32\% (PSNR 0) & 0.0212 & 0.3146 & 0.6016 & 0.6977 \\
28\% (PSNR 10) & 0.0885 & 0.7322 & 0.8231 & 0.8870 \\
$10 \%$ (PSNR 30) & 0.4638 & 0.9727 & 0.9547 & 0.9823 \\
2\% (PSNR 50) & 0.4945 & 0.9834 & 0.9580 & 0.9840 \\
\hline
\end{tabular}

Table 4. Comparisons of similarity measures for same images under Gaussian and slat and pepper noise

\begin{tabular}{ccccc}
\hline Ratio of noise & SSIM & $E M M$ & HFM & HFEMM \\
\hline 52\% (PSNR -30) & 0.0000 & 0.0118 & 0.3596 & 0.4695 \\
38\% (PSNR -10) & 0.0020 & 0.0039 & 0.3469 & 0.4599 \\
32\% (PSNR 0) & 0.0072 & 0.1050 & 0.463 & 0.5655 \\
28\% (PSNR 10) & 0.0290 & 0.2973 & 0.6280 & 0.7041 \\
8\% (PSNR 50) & 0.1045 & 0.7316 & 0.8430 & 0.8935 \\
1\% (PSNR 50) & 0.7295 & 0.9964 & 0.9849 & 0.9947 \\
65\% (PSNR-50) & 0.9774 & 0.9999 & 0.9996 & 1.0000 \\
\hline
\end{tabular}




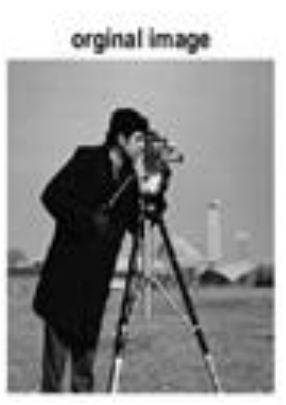

\section{Gaussian \& impluse}

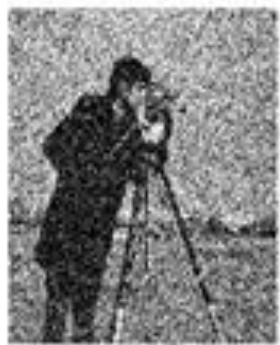

Figure 8. (P) original image and noisy image (q)

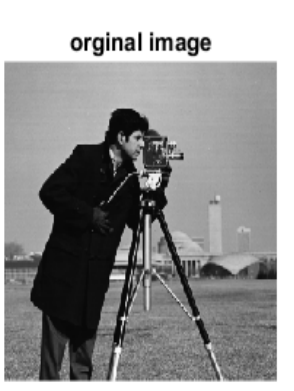

Gaussian+uniform-Noisy psnr=

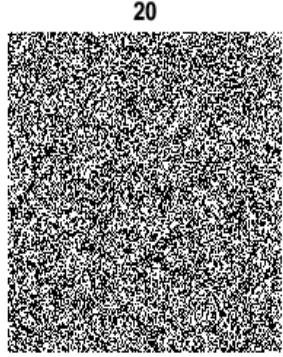

Figure 10. Original Image and noisy image

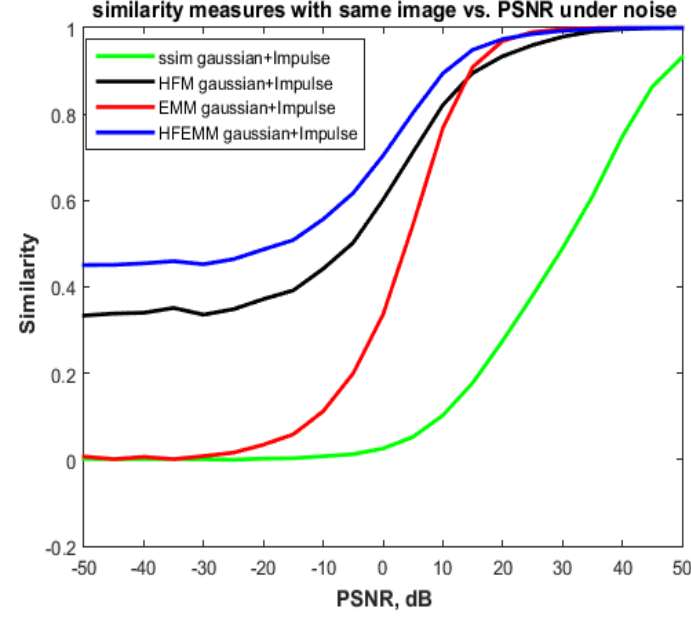

Figure 9. Comparisons of similarity measures under Gaussian and slat and pepper noise

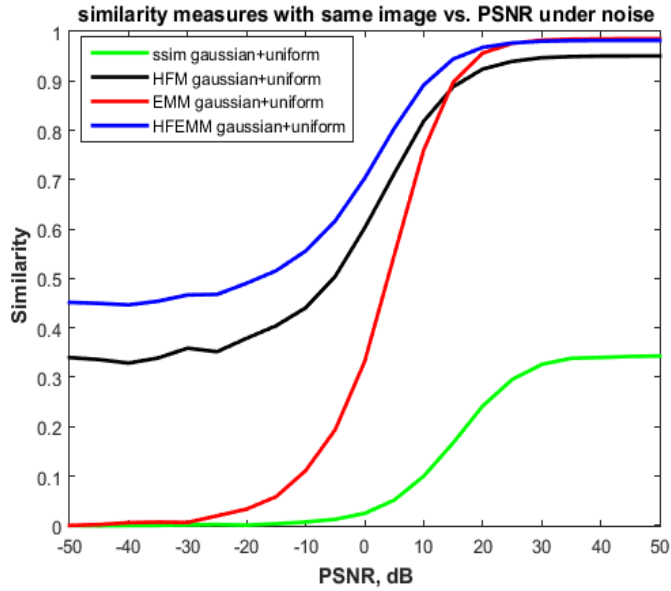

Figure 11. Comparisons of similarity measures under Gaussian and uniform noise

\section{CONCLUSION}

A hybrid similarity measure, called Histogram Feature Error Mean Measure, has been proposed. The proposed measure is depended on information theoretic features and statistical features. A joinit histogram' with original histogram have been used as information-theoretic tool, and Feature Measure with Error Mean have been used as a statistical tool. The proposed measure has been tested on $\mathrm{AT}$ and $\mathrm{T}$ and different types of images. We concluded that the new measure gave better performance (more similarity) than the other similarity measures such as (SSIM, EMM) under different kinds of noise when power of noise is highly high. The proposed measure can be used in a fundamental issue in real-world applications. Such as can be employed in found simlarity and different between image, verification, recognition (face, iris, and other pattern recognition systems).

\section{ACKNOWLEDGEMENTS}

We would like to thanks department of computer science in faculty of education for girlss in university of kufa, and Faculty of Computer Sciencec and inforbmation technology, University of Qadisiyah for their support. 


\section{REFERENCES}

[1] S. Farisa, C. Haviana, and D. Kurniadi, "Average Hashing for Perceptual Image Similarity in Mobile Phone Application," J. Telemat. Informatics, vol. 4, no. 1, pp. 12-18, 2016.

[2] D. Lin, "An Information-Theoretic Definition of Similarity," Icml, pp. 296-304, 1998.

[3] K. Shin, "An alternative approach to measure similarity between two deterministic transient signals," J. Sound Vib., vol. 371, pp. 434-445, 2016.

[4] A. F. Hassan, D. Cai-lin, and Z. M. Hussain, "An information-theoretic image quality measure: Comparison with statistical similarity,” J. Comput. Sci., vol. 10, no. 11, pp. 2269-2283, 2014.

[5] P. A. Viola, "massachusetts institute of technology alignment by Maximization of Mutual Information," Artif. Intell., no. 1548, 1995.

[6] Zhou Wang and I. Alan C. Bovik, Fellow, "Image Quality Assessment: From Error Visibility to Structural Similarity," IEEE Trans. Image Process., vol. 13, no. 4, pp. 1-14, 2004.

[7] A. F. Hassan, "An Information-Theoretic Measure for Face Recognition : Comparison with Structural Similarity," Int. J. Adv. Res. Artif. Intell., vol. 3, no. 11, pp. 7-13, 2014.

[8] R. A. Dihin and Z. M. Hussain, "Face Recognition over FFT-OFDM Computer Networks," Int. J. Appl. Eng. Res., vol. 12, pp. 14764-14773, 2017.

[9] Akash, O. M; Ahmad, Sharifah Sakinah Binti Syed; Azmi, Mohd Sanusi Bin, "A New Similarity Measure Based Affinity Propagation for Data Clustering," Advanced Science Letters, Volume 24, Number 2, pp. 1130-1133(4), February 2018.

[10] M. A. Aljanabi, N. A. Shnain, and S. F. Lu, "An image similarity measure based on joint histogram-Entropy for face recognition," 2017 3rd IEEE Int. Conf. Comput. Commun. ICCC 2017, vol. 2018-Janua, no. December, pp. 1626-1631, 2018.

[11] Mohammed Abdulameer Aljanabi, Huazhong and Wuhan, "Design of a hybrid measure for image similarity: a statistical, algebraic, and information-theoretic approach," European Journal of Remote Sensing, 2019.

[12] D. Brunet, E. R. Vrscay, and Z. Wang, "On the mathematical properties of the structural similarity index," IEEE Trans. Image Process . On, vol. 21, no. 4, pp. 1488-1499, 2012.

[13] R. Dosselmann and X. D. Yang, "A Formal Assessment of the Structural Similarity Index," Technical Report TRCS 2008-2 September, 2008.

[14] L. Zhang, L. Zhang, X. Mou, and D. Zhang, "FSIM: A feature similarity index for image quality assessment," IEEE Trans. Image Process., vol. 20, no. 8, pp. 2378-2386, 2011.

[15] U. Sara, M. Akter, and M. S. Uddin, "Image Quality Assessment through FSIM, SSIM, MSE and PSNR-A Comparative Study,” J. Comput. Commun., vol. 07, no. 03, pp. 8-18, 2019.

[16] F. M. Altufaili, "A Correlative Information-Theoretic Measure for Image Similarity," Global Journal of Pure and Applied Mathematics, vol. 13, no. 2, pp. 159-169, 2017.

[17] R. Soundararajan and A. C. Bovik, "Survey of information theory in visual quality assessment," Signal, Image Video Process., vol. 7, no. 3, pp. 391-401, 2013.

[18] T. Kalaiselvi, P. Sriramakrishnan, and K. Somasundaram, "Brain abnormality detection from MRI of human head scans using the bilateral symmetry property \& histogram similarity measures," Int. Comput. Sci. Eng. Conf., no. octoper 2016, pp. 1-6, 2016.

[19] N. R. Hamza, H. R. Mohammed, and Z. M. Hussain, "A joint histogram - 2D correlation measure for incomplete image similarity,” Int. J. Appl. Eng. Res., vol. 12, no. 22, pp. 12755-12761, 2017.

[20] Charles Boncelet, "Image Noise Models," In Alan C. Bovik. Handbook of Image and Video Processing. Academic Press, 2005.

[21] Abd Al-salam Selami, Ameen \& Fadhil, Ahmed. "A Study of the Effects of Gaussian Noise on Image Features," Kirkuk University Journal/Scientific Studies (1992-0849). 11. 152-169, 2016.

[22] F. M. Altufaili, H. R. Mohammed, Z.M. Hussain, "A Noise-Resistant Hybrid Measure for Images Similarity", 2016.

[23] Z. Wang and A. C. Bovik, "Mean squared error: love it or leave it? A new look at signal fidelity measures," IEEE Signal Processing Magazine, 2009.

[24] T. Kalaiselvi, P. Sriramakrishnan, and P. Nagaraja, "Brain Tumor Boundary Detection by Edge Indication Map Using Bi-Modal Fuzzy Histogram Thresholding Technique from MRI T2-Weighted Scans," Int. J. Image, Graph. Signal Process., vol. 8, no. 9, pp. 51-59, 2016.

[25] AT \& T Laboratories Cambridge, "AT \& T face database (formerly 'the ORL Database of Faces')", 2002, http://www.cl.cam.ac.uk/research/dtg/attarchive/ facedatabase.html 